\title{
Power Quality, Smart Meters and Additional Information from Different Power Terms
}

\author{
W. A. Souza, F. P. Marafão, Member, IEEE, E. V. Liberado, I. S. Diniz and P. J. A. Serni
}

\begin{abstract}
This paper shows how the detailed examination of active and nonactive power components may produce new information for modern smart meters. For this purpose, a prototype of electronic power meter has been implemented and applied to the evaluation of the Conservative Power Theory (CPT). Considering five sorts of loads, under four different operating conditions, the experimental results indicate that the CPT is able to provide a good methodology for load characterization, which could possibly benefits consumers and power utilities in several different ways. The results also show that depending on the situation, the analysis of nonactive power terms may be more important than the observation of traditional power quality indices, such as total harmonic distortion, unbalance factors or the fundamental positive sequence power factor.
\end{abstract}

Keywords- Conservative Power Theory, Power Meters, Power Quality, Revenue Metering, Smart Grids, Smart meters.

\section{INTRODUÇÃO}

A ÁREA de Qualidade de Energia Elétrica (QEE) tem se consolidado nas últimas duas décadas como campo de estudo de técnicas de monitoração, análise e mitigação de distúrbios da conformidade da energia elétrica. Em linhas gerais, a área de QEE estende-se desde preocupações com a operação de equipamentos sensíveis, até preocupações sobre a conservação da energia e eficiência das instalações.

Desta forma, foram criadas recomendações e normas de QEE em todo o mundo [1-10], estabelecendo condições e limites aceitáveis para assegurar a qualidade da energia. Obviamente, são necessárias ferramentas de análise e diagnóstico que permitam calcular os indicadores propostos, determinar as causas e possíveis consequências dos distúrbios na rede. Assim, diversas técnicas de processamento têm sido desenvolvidas, de forma a transformarem os dados de tensões e correntes medidas em informações úteis aos consumidores e concessionárias de energia [6,8,11-22].

Tais técnicas e metodologias de análise têm sido implementadas, tipicamente através dos chamados medidores

de qualidade de energia, os quais são utilizados quando um

W. A. Souza, Universidade Estadual de Campinas (UNICAMP), Campinas, São Paulo, Brasil, wesley@fc.unesp.br

F. P. Marafão, Universidade Estadual Paulista (UNESP), Sorocaba, São Paulo, Brasil, fmarafao@sorocaba.unesp.br

E. V. Liberado, Universidade Estadual de Campinas (UNICAMP), Campinas, São Paulo, Brasil, eduardoverriliberado@gmail.com

I. S. Diniz, Universidade Estadual Paulista (UNESP), Sorocaba, São Paulo, Brasil, ivando@sorocaba.unesp.br

P. J. A. Serni, Universidade Estadual Paulista (UNESP), Sorocaba, São Paulo, Brasil, serni@sorocaba.unesp.br problema de qualidade de energia manifesta-se em termos da operação indevida de equipamentos ou instalações. Entretanto, são poucas as instalações que dispõem de tais equipamentos para monitoramento permanente da QEE.

$\mathrm{Na}$ grande maioria das instalações industriais, os medidores eletrônicos disponíveis são capazes de fornecer informações sobre energia ativa e reativa (fator de potência), bem como armazenar detalhes sobre eventuais interrupções de energia. Já em instalações alimentadas em baixa tensão, tipicamente residenciais ou comerciais, é preponderante o uso dos tradicionais medidores de energia eletromecânicos, os quais permitem apenas a leitura (manual) do consumo de energia ativa [23-24].

No entanto, é crescente a tendência de que os medidores passem a fornecer maiores informações, sejam para as concessionárias de energia, sejam para os consumidores, bem como incorporar novas características que permitam, dentre outras funcionalidades, a comunicação com centrais de controle e supervisão. Tal tendência tem sido impulsionada nos últimos anos, principalmente, pelas discussões e pesquisas sobre Redes Inteligentes de Energia e Geração distribuída [14, 25-29].

Neste contexto, estão sendo desenvolvidos e implantados, em alguns países, modelos de medidores inteligentes com capacidade de: medição de energia em condições de fluxo bidirecional; comunicação com centrais de gerenciamento das concessionárias; tarifação horo-sazonal; conexão ou desconexão em função de sistema de pré-pagamento de conta; envio automático de valores de consumo para as concessionárias, seja horário ou diariamente; ou ainda, capacidade de medição integrada com outros bens de consumo (e.g., gás natural) [30-38].

Dentre muitas novas características, a literatura internacional tem trazido discussões sobre formas de comunicação entre os medidores e consumidores ou concessionárias (Zigbee, IEEE $802.11 \mathrm{~b} / \mathrm{g}$, PLC - power line communication, Bluetooth, etc.), bem como formas de apresentação das informações detalhadas do consumo [32-36]. No entanto, algumas questões importantes no contexto da medição e tarifação de energia, principalmente aquelas associadas ao fator de potência e à QEE não tem sido amplamente abordadas. Pouco se tem discutido sobre prover o consumidor de informações que o permitam melhor gerenciar ou confrontar seu consumo de energia e a qualidade de energia com sua respectiva concessionária.

Corre-se o risco de que um grande número de medidores de energia seja substituído nos próximos anos, os quais ainda não 
permitirão a monitoração de indicadores consolidados de QEE ou mesmo, a atualização de seus códigos com futuras metodologias de análise da energia elétrica que beneficiem tanto concessionárias, quanto consumidores de energia.

Neste contexto, as discussões deste artigo vão além da criação de um protótipo de medidor inteligente, o qual permite a monitoração das grandezas elétricas e envio de informações para uma central de supervisão. É proposto que o medidor utilize uma metodologia recente de decomposição da potência elétrica em diversas sub-componentes, as quais permitem uma análise detalhada da carga, permitindo assim a descrição de fenômenos relevantes tanto do ponto de vista do consumidor, quanto das concessionárias de energia.

Tal formulação, conhecida por Teoria de Potência Conservativa (CPT, do inglês Conservative Power Theory), foi proposta por Tenti et. al. [39,40] e tem se mostrado uma interessante ferramenta para a interpretação de fenômenos físicos que caracterizam as instalações ou cargas elétricas [41,42], bem como para a mitigação de distúrbios [43-44], tarifação ou atribuição de responsabilidades [45-46].

Assim, as próximas seções apresentarão o protótipo de medidor inteligente desenvolvido, bem como uma descrição sucinta da CPT e resultados experimentais de sua aplicação, a fim de demonstrar sua utilidade como possível metodologia de análise da energia nos medidores eletrônicos do futuro.

\section{MEDIDOR ELETRÔNICO DESENVOLVIDO}

Com o intuito de implementar um protótipo de medidor inteligente para o estudo de possíveis metodologias de análise e tarifação da energia elétrica, as seguintes características foram assumidas:

- 12 canais de entrada analógica (4 tensões, 4 correntes e 4 temperaturas) com conversão analógico-digital (AD) de ao menos 12 bits;

- Taxa de amostragem igual ou superior a $6 \mathrm{kHz}$ por canal (4 vezes superior a $25^{\mathrm{a}}$ harmônica de $60 \mathrm{~Hz}$ );

- Tensão de entrada máxima de 215V (pico);

- Corrente de entrada máxima de 35A (pico);

- Tempo de gravação: de oito horas a 1200 dias, dependendo do intervalo de armazenagem.

A Fig. 1 indica o diagrama de blocos do medidor desenvolvido.

\section{A. Aquisição de dados}

$\mathrm{Na}$ parte correspondente à captura dos sinais de tensões e correntes, um circuito de sensoriamento e condicionamento dos sinais foi elaborado de forma a adequar as grandezas medidas aos níveis aceitáveis de conversão analógica-digital (AD) no DSP escolhido como núcleo de processamento do medidor (F28335 da Texas Instruments).

Concomitantemente à aquisição dos sinais de tensões e correntes, foram colocados quatro sensores de temperatura (modelo LM35DZ) no protótipo, com o propósito de ajuste dos sinais medidos pelos sensores (modelos LV25P e LA55P) em função da temperatura (média) no interior do medidor. Os ajustes foram programados através das curvas características fornecidas pelo fabricante dos sensores, em função da variação da temperatura para uma faixa de operação entre $0^{\circ} \mathrm{C}$ a $70^{\circ} \mathrm{C}$.

\section{B. Armazenamento dos dados}

No caso de armazenamento local, o DSP permite que seja utilizada a memória expandida, chamada de XINTF(), a qual tem capacidade de $4 \mathrm{MB}$. Cada pacote de dados armazenados corresponde a 144 bytes, o que resulta na estimativa de armazenamento descrita na Tabela 1, a partir da qual é possível verificar que, por exemplo, coletando as informações a cada minuto, é possível armazenar na memória local até 20 dias.

\section{Comunicação sem fio}

O medidor desenvolvido também pode enviar os dados para outros equipamentos do sistema de monitoração ou supervisão, se uma rede IEEE $802.11 \mathrm{~b} / \mathrm{g}$ estiver disponível. Para isto, foi utilizado um conversor UART para o padrão IEEE $802.11 \mathrm{~b} / \mathrm{g}$, comumente conhecido como padrão WiFi e, juntamente a este conversor, foi criado um protocolo baseado

TABELA I. ESTIMATIVA DO ARMAZENAMENTO LOCAL DO MEDIDOR.

\begin{tabular}{ll}
\hline Tempo de cada amostra & Tempo total de armazenamento \\
\hline 1 segundo & $\approx 8$ horas \\
1 minuto & $\approx 20$ dias \\
30 minutos & $\approx 600$ dias \\
1 hora & $\approx 1200$ dias
\end{tabular}

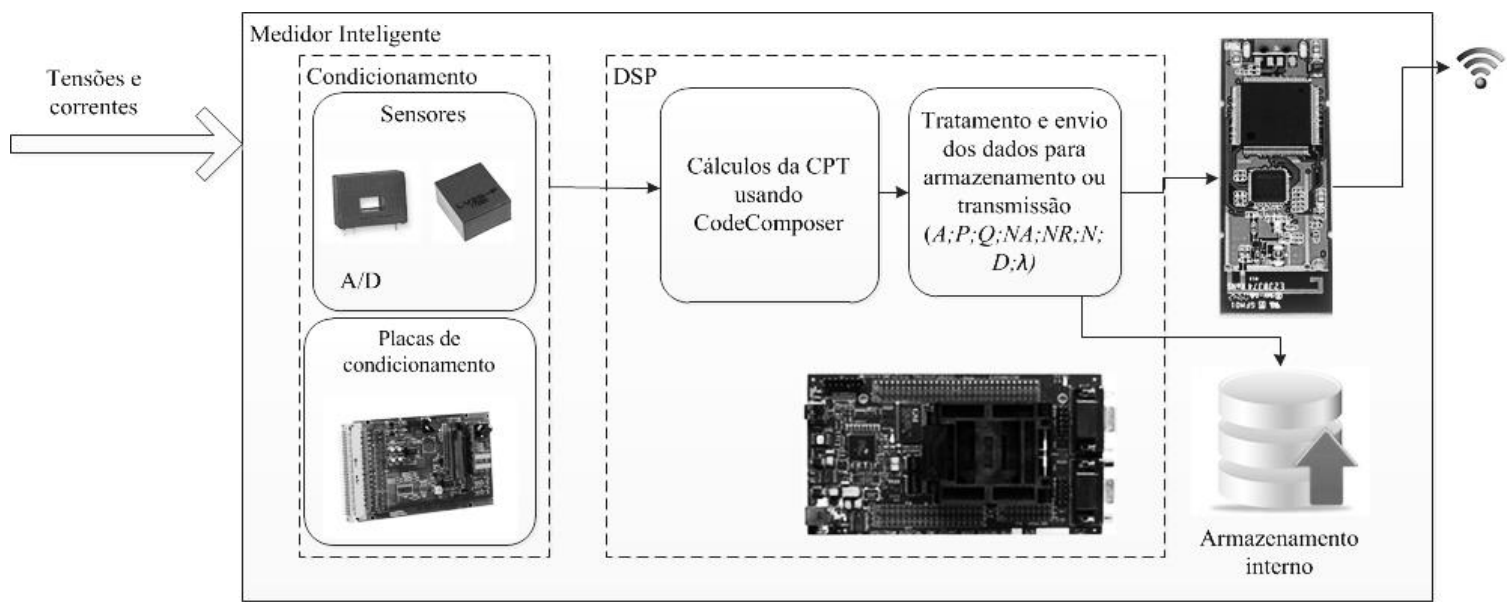

Figura 1. Diagrama de blocos do protótipo de medidor inteligente desenvolvido. 
em TCP/IP, o qual possibilita a comunicação entre dois dispositivos em WiFi usando conexão ad-hoc.

Assim sendo, os indicadores e variáveis calculadas são enviados serialmente pelo DSP. O conversor os transmite em padrão wireless e um computador receptor interpreta os dados enviados sob o protocolo criado e os armazena.

\section{Descrição geral do medidor}

A Fig. 2 apresenta algumas fotos do protótipo de medidor desenvolvido. Basicamente, o conjunto de hardware utilizado é constituído de:

- Fontes de tensão de $\pm 5 \mathrm{~V}$ e $\pm 15 \mathrm{~V}$;

- Placa de condicionamento de corrente;

- Placa de condicionamento de tensão;

- Kit eZdsp F28335;

- Módulo UART/Wifi Linksprite com antena Pigtail.

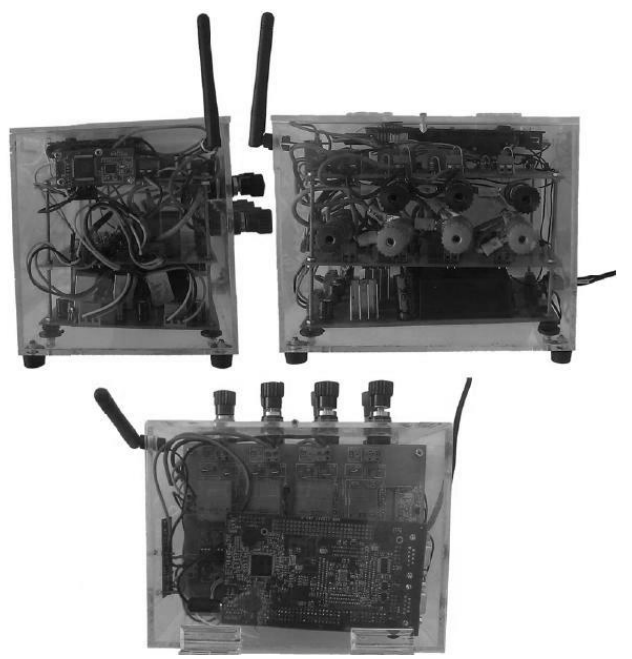

Figura 2. Fotos do protótipo de medidor desenvolvido.

\section{SÍNTESE DA TEORIA DE PotÊnCIA CONSERVATIVA}

A CPT é uma proposta recente de teoria de potência no domínio do tempo, a qual define grandezas elétricas chamadas de conservativas, as quais são calculadas a partir do produto escalar entre tensões e correntes (ou suas integrais imparciais) [40,41]. Tais tensões e correntes, assim como suas integrais imparciais, satisfazem as leis de tensões e correntes de Kirchhoff e, consequentemente, o teorema de Tellegen (Teorema da Conservação de Energia).

Assim, são definidas duas grandezas conservativas principais:

A potência ativa instantânea, a qual é expressa por meio do produto escalar:

$$
p(t)=\boldsymbol{v} \circ \boldsymbol{i}
$$

e a energia reativa instantânea expressa por:

$$
w(t)=\widehat{\boldsymbol{v}} \circ \boldsymbol{i}
$$

Sendo que:

$\boldsymbol{v}$ corresponde ao vetor de tensões de fase de um sistema $\mathrm{m}$ fásico $\left[\begin{array}{llll}v_{a} & v i_{b} & \ldots & v_{m}\end{array}\right]$;

$\boldsymbol{i}$ corresponde ao vetor de correntes de fase $\left[\begin{array}{llll}i_{a} & i_{b} & \ldots & i_{m}\end{array}\right]^{T}$;

$\widehat{\boldsymbol{v}}$ corresponde ao vetor contendo as integrais imparciais das tensões de fase .
A integral imparcial é definida como:

$$
\widehat{\boldsymbol{v}}_{m}=\boldsymbol{v}_{m}-\overline{\boldsymbol{v}}_{m} \text {, }
$$

com:

$\boldsymbol{v}_{m \int}=\int_{0}^{T} \boldsymbol{v}(t) d t$ sendo a integral no tempo e;

$\overline{\boldsymbol{v}}_{m \int}=\frac{1}{T} \int_{0}^{T} \boldsymbol{v}_{m \int} d t$ sendo o valor médio da integral no tempo.

Os valores médios de (1) e (2), respectivamente, a potência ativa média $(P)$ e a energia reativa média $(W)$, são indicados em (4) e (5):

$$
\begin{aligned}
& \boldsymbol{P}=\overline{\boldsymbol{p}}=\frac{1}{T} \int_{0}^{T} \boldsymbol{v}(t) \circ \boldsymbol{i}(t) d t \\
& \boldsymbol{W}=\overline{\boldsymbol{w}}=\frac{1}{T} \int_{0}^{T} \widehat{\boldsymbol{v}}(t) \circ \boldsymbol{i}(t) d t
\end{aligned}
$$

\section{A. Componentes de corrente da CPT}

Com base nas definições de potência ativa (4) e energia reativa (5), a CPT propõe uma decomposição da corrente em parcelas ortogonais que visam representar os fenômenos físicos (características do circuito equivalente) provocados pelas cargas do circuito (elementos lineares e não lineares, desbalanços e reatividade). Tais parcelas são descritas a seguir:

corrente ativa $\left(i_{a}\right)$ : é aquela responsável por transferir a mínima potência ativa por fase no circuito;

corrente reativa $\left(i_{r}\right)$ : é aquela responsável pelo fluxo mínimo de energia reativa por fase no circuito;

corrente residual ou nula $\left(i_{v}\right)$ : é aquela que não transfere potência ativa nem energia reativa (não linearidades do circuito);

Estas parcelas da corrente são denominadas de componentes básicas e são ortogonais entre si, ou seja, o produto escalar médio entre elas é nulo. Sendo assim, é possível definir a relação entre os valores eficazes dessas parcelas e o valor eficaz da corrente total:

$$
I^{2}=I_{a}^{2}+I_{r}^{2}+I_{v}^{2} .
$$

No caso de circuitos polifásicos (com ou sem condutor de retorno), as parcelas de corrente ativa e reativa podem ainda ser decompostas em parcelas balanceadas e desbalanceadas:

a corrente ativa balanceada $\left(i_{a}^{b}\right)$ é aquela responsável pela transferência mínima da potência ativa coletiva da fonte para a carga, além de ser proporcional e estar em fase com a tensão;

a corrente reativa balanceada $\left(i_{r}^{b}\right)$ é aquela responsável pelo fluxo mínimo de energia reativa coletiva no circuito e é ortogonal com relação à tensão medida. Vale destacar que tal corrente pode ser distorcida, caso a tensão o seja;

a corrente de desbalanço $\left(I^{u^{2}}=I_{a}^{u^{2}}+I_{r}^{u^{2}}\right)$ é composta por uma parte ativa e outra reativa, as quais representam os diferentes valores de condutância e de elementos reativos equivalente por fase.

Nesse sentido (6) pode ser expandida como:

$$
\boldsymbol{I}^{2}=\boldsymbol{I}_{a}^{2}+\boldsymbol{I}_{r}^{2}+\boldsymbol{I}_{v}^{2}=\boldsymbol{I}_{a}^{b^{2}}+\boldsymbol{I}_{r}^{b^{2}}+\underbrace{\boldsymbol{I}_{a}^{u^{2}}+\boldsymbol{I}_{r}^{u^{2}}}_{I^{u^{2}}}+\boldsymbol{I}_{v}^{2}
$$

Em (7) os valores das correntes são eficazes coletivos, ou seja, no cálculo do valor eficaz (RMS) de cada uma é considerada a soma dos quadrados das correntes de fase do circuito. Também neste caso, a ortogonalidade entre cada componente de corrente é mantida. 
Com a decomposição das correntes, também é possível encontrar parcelas de potência ortogonais entre si, as quais permitem uma descrição detalhada das características da carga.

\section{B. Componentes de potência da CPT}

Para cada parcela de corrente está associada uma potência, obtida pelo produto entre o valor eficaz da tensão e o valor eficaz da corrente correspondente:

Potência ativa $\left(\boldsymbol{P}=\boldsymbol{V} \boldsymbol{I}_{a}^{b}\right)$ : é aquela tida como o consumo médio de potência do circuito, representando a realização de trabalho útil na carga;

Potência reativa $\left(\boldsymbol{Q}=\boldsymbol{V} \boldsymbol{I}_{r}^{b}\right)$ : está relacionada à defasagem entre tensões e correntes, a qual pode ser provocada pela presença de elementos armazenadores de energia ou por cargas eletrônicas;

Potência de desbalanço $\left(\boldsymbol{N}=\boldsymbol{V} \sqrt{\boldsymbol{I}_{a}^{u^{2}}+\boldsymbol{I}_{r}^{u^{2}}}\right)$ : é a parcela de potência que representa a presença de cargas desbalanceadas entre as fases;

Potência de distorção $\left(\boldsymbol{D}=\boldsymbol{V} \boldsymbol{I}_{v}\right)$ : é a parcela de potência consumida devido à presença de não linearidades no circuito. Esta não realiza trabalho útil, nem caracteriza o armazenamento de energia;

Potência aparente $(\boldsymbol{A}=\boldsymbol{V I})$ é potência total do circuito, a qual pode ser decomposta a partir de (7), através da multiplicação dos termos quadráticos de corrente, pelo quadrado do valor eficaz da tensão:

$$
A^{2}=V^{2} I^{2}=P^{2}+Q^{2}+N^{2}+D^{2} .
$$

A CPT propõe ainda um fator de potência polifásico (global), calculado como em (9):

$$
\lambda=\frac{P}{\boldsymbol{A}}=\frac{\boldsymbol{I}_{a}^{b}}{\boldsymbol{I}}
$$

Este fator de potência relaciona a corrente ativa balanceada com todas as outras parcelas de corrente, através da corrente coletiva total, e resulta unitário somente no caso de circuito puramente resistivo balanceado. Do contrário, a presença de elementos armazenadores de energia, desbalanço da carga e não linearidades podem influenciar no fator de potência.

De (9), destaca-se que de acordo com a definição da corrente ativa balanceada (proporcional à tensão), uma carga resistiva balanceada, mesmo que alimentada por tensões não senoidais e/ou assimétricas, pode resultar em fator de potência unitário.

Vale observar que de acordo com a CPT, o cálculo das potências apresentadas pode ser realizado mesmo em condições com formas de onda distorcidas e/ou desequilibradas, inclusive a potência reativa, diferentemente de grande parte das abordagens tradicionais [47] ou mesmo da recente recomendação do IEEE [15].

Maiores detalhes sobre o equacionamento e as propriedades dos operadores matemáticos associados às definições da Teoria de Potência Conservativa podem ser encontrados em [48].

\section{RESULTADOS EXPERIMENTAIS}

Buscando demonstrar como uma metodologia de decomposição da potência em suas parcelas ativa e não ativas poderia ser utilizada para a interpretação dos circuitos elétricos e consecutivamente, justificando sua implementação nos medidores inteligentes do futuro, as próximas seções descrevem o conjunto de casos analisados.

\section{A. Descrição dos casos analisados}

O diagrama simplificado do circuito utilizado pode ser visto na Figura 3. O medidor foi alocado no PAC e uma fonte de tensão programável foi utilizada para gerar as diferentes configurações de tensão, conforme descrição da Tabela II (casos de A-D). A Tabela III apresenta as diferentes configurações de cargas que foram analisadas (casos de 1-6).

Os resultados são apresentados nas Tabelas IV e V e, com a exceção dos casos apresentados com “*”, os circuitos são trifásicos a quatro fios, sem impedância de linha. Os casos indicados com “*” representam circuitos trifásicos sem condutor de retorno (três fios). Com a finalidade de comparação, os valores na última linha das Tabelas IV e V indicam o fator de potência de sequência positiva, calculados conforme proposto em [15].

Os valores cuja proporção em relação ao maior valor medido é menor que $0,1 \%$ foram omitidos da tabela, sendo denotados por "-".

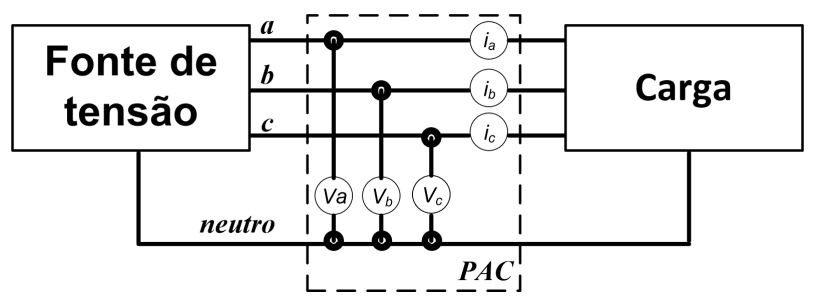

Figura 3. Configuração básica do circuito usado no experimento.

TABELA II. CONFIGURAÇÕES DE TENSÕES.

Descrição da Tensão

\begin{tabular}{ll}
\hline A & Senoidal e simétrica (127VRMS em cada fase). \\
B & Não senoidal e simétrica (127VRMS adicionadas as $3^{\mathrm{a}}, 5^{\mathrm{a}}, 7^{\mathrm{a}} \mathrm{e}$ \\
& $9^{\mathrm{a}}$ harmônicas, sendo cada harmônica correspondente a $5 \%$ da \\
& tensão fundamental). \\
C & Senoidal e assimétrica (Va=127VRMS, Vb=113VRMS e \\
& Vc=135VRMS). \\
D & Não senoidal e assimétrica (Va=127VRMS, Vb=113VRMS e \\
& Vc=135VRMS, adicionadas as $3^{\mathrm{a}}, 5^{\mathrm{a}}, 7^{\mathrm{a}}$ e $9^{\mathrm{a}}$ harmônicas, \\
& sendo cada harmônica correspondente a $5 \%$ da tensão \\
fundamental de cada fase).
\end{tabular}

TABELA III. CONFIGURAÇÕES DAS CARGAS

Descrição das cargas

\begin{tabular}{ll}
\hline 1 & Resistiva balanceada $(\mathrm{R}=8,5 \Omega$ em cada fase $), \mathrm{em} \mathrm{Y}$. \\
2 & $\begin{array}{l}\text { Resistiva desbalanceada }(\mathrm{Ra}=11.8 \Omega ; \mathrm{Rb}=5.9 \Omega ; \mathrm{Rc}=8.5 \Omega), \mathrm{em} \\
\mathrm{Y} .\end{array}$ \\
3 & $\begin{array}{l}\text { Resistiva-indutiva balanceada }(\mathrm{R}=8.5 \Omega ; \mathrm{L}=15 \mathrm{mH} \text { em cada } \\
\text { fase }), \text { em } \mathrm{Y} .\end{array}$ \\
& Resistiva-indutiva desbalanceada $(\mathrm{Ra}=11,8 \Omega ; \mathrm{Rb}=5,9 \Omega ;$ \\
& Rc $=8,5 \Omega ; \mathrm{La}=20 \mathrm{mH} ; \mathrm{Lb}=10 \mathrm{mH} ; \mathrm{Lc}=15 \mathrm{mH})$, em $\mathrm{Y}$. \\
5 & $\begin{array}{l}\text { Não linear balanceado }(\mathrm{LNL}=0,5 \mathrm{mH} \text { por fase; retificador } \\
\text { trifásico a diodos; } \mathrm{CNL}=2,35 \mathrm{mF} ; \mathrm{RNL}=13,8 \Omega)\end{array}$
\end{tabular}


TABELA IV. RESUlTADOS EXPERIMENTAIS 1 - COMPONENTES DE POTÊNCIA E FATOR DE POTÊNCIA DA CPT.

\begin{tabular}{lcccccccccccc}
\hline \hline & $\mathrm{A} 1$ & $\mathrm{~A} 2$ & $\mathrm{~A} 2 *$ & $\mathrm{~A} 3$ & $\mathrm{~A} 4$ & $\mathrm{~A} 5$ & $\mathrm{~B} 1$ & $\mathrm{~B} 2$ & $\mathrm{~B} 2 *$ & $\mathrm{~B} 3$ & $\mathrm{~B} 4$ & $\mathrm{~B} 5$ \\
\hline $\mathrm{A}[\mathrm{VA}]$ & 5848 & 6239 & 5782 & 4812 & 4927 & 6942 & 5746 & 6321 & 5925 & 4773 & 4965 & 7022 \\
$\mathrm{P}[\mathrm{W}]$ & 5849 & 6009 & 5617 & 4011 & 4099 & 6112 & 5746 & 6085 & 5815 & 3965 & 4075 & 6045 \\
$\mathrm{Q}$ [VA] & - & - & - & 2541 & 2583 & 802,2 & - & - & - & 2644 & 2649 & 1674 \\
$\mathrm{Na}$ [VA] & - & 1645 & 785,54 & - & 667,3 & - & - & 1712 & 803,68 & - & 688,5 & - \\
$\mathrm{Nr}[\mathrm{VA}]$ & - & - & 830,96 & - & 700,8 & - & - & - & 801,63 & - & 690,1 & - \\
$\mathrm{N}[\mathrm{VA}]$ & - & 1660 & 1143 & - & 983,8 & - & - & 1712 & 1135 & - & 974,8 & - \\
$\mathrm{D}[\mathrm{VA}]$ & - & - & - & - & - & 3111 & - & - & - & 263,20 & 274,7 & 3158 \\
$\lambda$ & 0,9967 & 0,9659 & 0,9678 & 0,8300 & 0,8271 & 0,8829 & 1,000 & 0,9630 & 0,9810 & 0,8310 & 0,8210 & 0,8610 \\
$\mathrm{FP}_{1}{ }^{+}[15]$ & 0,9982 & 0,9981 & 0,9937 & 0,8304 & 0,7681 & 0,9331 & 1,000 & 0,9922 & 0,9937 & 0,8325 & 0,8394 & 0,9635
\end{tabular}

TABELA V. RESUltados EXPERIMENTAIS 2 - COMPONENTES DE POTÊNCIA E FATOR DE POTÊNCIA DA CPT.

\begin{tabular}{lllllll}
\hline \hline & C1 & C3 & C5 & D1 & D3 & D5 \\
\hline $\mathrm{A}[\mathrm{VA}]$ & 5663 & 4720 & 6663 & 5652 & 4678 & 6268 \\
$\mathrm{P}[\mathrm{W}]$ & 5662 & 3944 & 5735 & 5636 & 3899 & 5030 \\
$\mathrm{Q}[\mathrm{VA}]$ & - & 2527 & 893,8 & - & 2450 & 904,2 \\
$\mathrm{Na}[\mathrm{VA}]$ & - & - & 629,6 & - & - & 1208 \\
$\mathrm{Nr}[\mathrm{VA}]$ & - & - & 1049 & - & - & 1261 \\
$\mathrm{~N}[\mathrm{VA}]$ & - & - & 1223 & - & - & 1746 \\
$\mathrm{D}[\mathrm{VA}]$ & - & - & 3047 & - & 238,8 & 3079 \\
$\lambda$ & 0,9995 & 0,8343 & 0,8679 & 0,9994 & 0,8322 & 0,8072 \\
$\mathrm{FP}_{1}^{+}[15]$ & 0,9987 & 0,8325 & 0,9582 & 0,9986 & 0,8325 & 0,9452
\end{tabular}

\section{B. Análise dos resultados}

Casos A1, B1, C1 e D1-Carga resistiva balanceada

Sabidamente, este tipo de circuito representa o tipo ideal de carga para o sistema elétrico, no qual toda a potência elétrica fornecida é transformada em trabalho útil. Isto significa que este circuito só absorve potência ativa $(\mathrm{P})$, resultando em fator de potência unitário para quaisquer que sejam as formas de onda das tensões de fornecimento.

Entretanto, é interessante observar como tal circuito poderia ser interpretado através da CPT, especialmente nos casos B1, C1 e D1, nos quais as tensões de alimentação são distorcidas e/ou assimétricas (Tabela II). É possível observar que além de não indicar a presença de reativos $(\mathrm{Q})$, a CPT também não indica a presença de potência de desbalanço (N) ou de distorção (D), mesmo nos casos de tensões deterioradas. Isto significa dizer que qualquer distorção ou desequilíbrio nas correntes da carga não foi imposto pelo comportamento da carga, mas sim das tensões de alimentação.

Conclusão semelhante dificilmente poderia ser extraída se a análise fosse feita através dos indicadores convencionais de QEE, por exemplo, Distorção Harmônica Total (DHT) ou fatores de desequilíbrio $\left(\mathrm{K}^{-}, \mathrm{K}^{0}\right)$. Provavelmente, a observação de tais fatores indicaria a presença de cargas não lineares e/ou desbalanceadas nos casos citados.

\section{Casos A2, B2 - Carga resistiva desbalanceada}

Em ambos os casos, além da potência ativa (P), a CPT indica a presença de potência de desbalanço ativa $(\mathrm{Na})$, a qual é provocada pelo desbalanço entre as condutâncias de fase do circuito. Vale ressaltar que no caso B2, mesmo as tensões sendo distorcidas, a metodologia não indica a presença de potência de distorção (D). Por outro lado, o desbalanço da carga representa uma característica não desejada, a qual provoca a redução da eficiência do sistema, sendo tal efeito destacado pela redução do fator de potência.

Este caso ressalta a contribuição do desbalanço de carga na redução do fator de potência, efeito este pouco discutido quando se trata da definição de fator de potência trifásico nos medidores eletrônicos modernos.

Além disto, dependendo da abordagem escolhida para o cálculo do fator de potência [15, 47], os valores obtidos podem ser muito distintos. Vide $\mathrm{FP}_{1}^{+}$na Tabela IV, o qual resulta significativamente maior nos casos $\mathrm{A} 2$ e $\mathrm{B} 2$.

\section{Casos $A 2 *, B 2 *$ - Carga resistiva desbalanceada a três fios}

Diferentemente do caso anterior, nos casos com "*" o circuito não possui condutor de retorno. Isto significa que o desbalanço da carga provoca desequilíbrio nas tensões sobre os terminais da carga (o que não ocorre no caso com condutor de retorno).

Nesta situação, apesar de não indicar a presença de potência reativa (Q) ou de distorção (D), a CPT indica a existência de potências de desbalanço ativa $(\mathrm{Na})$ e reativa $(\mathrm{Nr})$. Apesar de curiosa, a indicação da potência de desbalanço reativa é justificada pela defasagem entre as tensões e correntes no caso do circuito a três fios com carga desbalanceada.

Estes casos revelam que a CPT é capaz de caracterizar condições de operação bastante específicas, como a defasagem entre tensões e correntes provocada não pela presença de armazenadores de energia, mas pelo deslocamento da tensão de neutro da carga em Y desbalanceada.

Casos A3, B3, C3 e D3 - Carga Resistivo-Indutiva Balanceada

Nestes casos, a presença da carga de comportamento indutivo resulta na ocorrência de potência reativa (Q) e consequente redução do fator de potência.

Vale observar que sendo a carga balanceada, a potência de desbalanço não é indicada em nenhuma condição de operação, 
mesmo com tensões desequilibradas (C3 e D3). No entanto, a existência de tensões distorcidas nos casos B3 e D3 resultam no surgimento de potência de distorção (D). A potência de distorção nestes casos está associada ao fenômeno de dispersão harmônica (scattering effect), o qual representa (simplificadamente) a diferença entre as formas de onda de tensão e corrente, provocada pelo comportamento da carga indutiva sendo alimentada por tensões distorcidas. Tal fenômeno já foi discutido por outros autores [49] e detalhes de sua interpretação através da CPT podem ser encontrados em $[39,48]$.

\section{Casos A4, B4-Carga resistivo-indutiva desbalanceada}

Nesta configuração de carga, a CPT indica a presença de potências reativa e de desbalanço (ativa e reativa), sejam as tensões de alimentação senoidais ou distorcidas. A potência de desbalanço reativa neste caso é relacionada com o comportamento desbalanceado da carga RL, uma vez que se trata de circuito com condutor de retorno. Novamente, no caso B4 a metodologia destaca o fenômeno de dispersão através da potência de distorção. Nestes casos, Q, N e D contribuem para a redução do fator de potência.

\section{Casos A5, B5, C5 e D5 - Carga Não Linear balanceada}

A análise de circuitos com cargas não lineares através da CPT permite conclusões interessantes. Nos casos A5 e B5, a CPT indica apenas a existência das potências ativa $(\mathrm{P})$, de distorção (D, proveniente da não linearidade da carga) e reativa $(\mathrm{Q}$, proveniente do filtro indutivo de entrada do retificador).

Nos casos C5 e D5, além das parcelas mencionadas a CPT também indica a presença de potência de desbalanço $(\mathrm{N})$. Este resultado poderia indicar uma contradição com relação, por exemplo, as conclusões extraídas dos casos C1 e D1 que indicaram que a carga resistiva balanceada não é afetada pelos desequilíbrios das tensões. Entretanto, a análise das correntes de fase indica que a carga não linear tem seu comportamento severamente alterado, se alimentada por tensões desequilibradas.

Portanto, a análise destes quatro casos indica que o comportamento de cargas não lineares pode ser alterado significativamente pelas tensões de fornecimento, afetando inclusive o fator de potência, o qual neste caso variou entre 0,88 e 0,80 .

Também é interessante observar a diferença entre os fatores de potência calculados pela CPT $(\lambda)$ e aquele proposto em [15]. Para todas as condições de operação analisadas com a carga não linear (A5, B5, C5 e D5), enquanto a CPT indicaria pela penalização do fator de potência, os valores do fator de potência de sequência positiva estariam acima do valor de referência no Brasil $(0,92)$.

\section{CONCLUSÕES}

Este artigo apresentou a descrição básica do protótipo de medidor inteligente desenvolvido e utilizado para avaliação da Teoria de Potência Conservativa, na interpretação de diferentes configurações de circuitos elétricos.
A avaliação da CPT indicou que a utilização de uma teoria de potência que permita a decomposição da potência em diversas sub parcelas pode agregar informações importantes para a caracterização do comportamento das cargas ou instalações elétricas. Tais informações poderiam auxiliar concessionárias e/ou consumidores na definição de estratégias de gerenciamento de energia, compensação de distúrbios, bem como na definição de metodologias de atribuição de responsabilidades.

Do ponto de vista de atribuição de responsabilidades, na maioria dos casos analisados, a CPT foi capaz de caracterizar a carga em questão, independentemente da tensão de fornecimento. Entretanto, no caso de circuito com carga não linear e tensões de fornecimento desequilibradas, as tensões afetam significativamente a operação da carga não linear, o que dificulta desassociar as características da carga e da fonte de tensão.

Entretanto, ainda que estudos adicionais e novas metodologias de atribuição de responsabilidades ou tarifação ainda precisem ser desenvolvidas ou aprimoradas, a análise de alguns dos casos estudados indicou que a utilização de uma metodologia como a CPT pode ser mais interessante para a caracterização das cargas, do que a simples aplicação de indicadores tradicionais de qualidade de energia.

Finalizando, os autores acreditam que as discussões e proposições sobre os chamados "medidores inteligentes do futuro" não poderiam deixar de contemplar as discussões sobre cálculos de potência e fator de potência em circuitos polifásicos não lineares. Neste contexto, destaca-se o papel fundamental das agências reguladoras no sentido de definirem características não apenas do hardware ou protocolos de comunicação dos medidores, mas também dos algoritmos que estes deverão usar para caracterizar o consumo (ou geração) de energia nas instalações, além de eventuais metodologias de atribuição de responsabilidades que venham a ser definidas nos próximos anos.

\section{AGRADECIMENTOS}

Os autores agradecem à FAPESP (Fundação de Amparo à Pesquisa do Estado de São Paulo), processo 2009/04918-7, pelo financiamento do projeto.

\section{REFERÊNCIAS}

[1] ANEEL, "Procedimentos de Distribuição de Energia Elétrica no Sistema Elétrico Nacional - PRODIST", Módulos de 1 a 8, Agência Nacional de Energia Elétrica, 2012.

[2] ANEEL, "Resolução Normativa n $n^{\circ} 414$ de 9 de Setembro de 2010", Agência Nacional de Energia Elétrica, 2010.

[3] IEEE, Draft Guide for Applying Harmonic Limits on Power Systems, IEEE P519.1/D12, pp.1,124, Jul 2012.

[4] IEC, International Standard 61000-3-2: Electromagnetic compatibility (EMC) - Limits on harmonics current emissions, Nov 2005.

[5] IPQ, "Características da tensão fornecida pelas redes de distribuição pública de energia eléctrica", Norma Portuguesa NP EN 50160 - 1995, Instituto Português da Qualidade, Feb 1996.

[6] IEC, IEC 61000-4-30 standard, "Electromagnetic compatibility (EMC) Part 4-30: Testing and measurement techniques - Power quality measurement methods", pp. 81, Oct 2008.

[7] EN 50160:1999 standard, "Voltage characteristics of electricity supplied by public distribution systems", Feb 2010. 
[8] V. Ajodhia and B. Franken, "Regulation of Voltage Quality", Workpackage 4 and 5 from project Quality of Supply and Regulation, KEMA Connsulting, Feb 2007.

[9] C. Masetti, "Revision of European Standard EN 50160 on power quality: Reasons and solutions", 14th International Conference on Harmonics and Quality of Power, pp.1,7, Sept 2010.

[10] A. Broshi, "Monitoring power quality beyond EN 50160 and IEC 610004-30", 9th International Conference on Electrical Power Quality and Utilisation, vol., no., pp.1-6, Oct 2007.

[11] J. de-la-Rosa and A. Moreno-Muñoz, "A web-based distributed measurement system for electrical Power Quality monitoring”, IEEE Sensors Applications Symposium, pp. 206-211, Feb 2010.

[12] B. Byman, T. Yarborough, R. S. Von Carolsfeld and J. Van Gorp, "Using distributed power quality monitoring for better electrical system management", Pulp and Paper Industry Technical Conference, pp. 21-26, Jun 1998.

[13] A. Ferrero, L. Peretto and R. Sasdelli, "Revenue metering in the presence of distortion and unbalance: myths and reality", IEEE International Conference on Harmonics and Quality of Power Proceedings, pp. 42-47, Oct 1998.

[14] P. Corral, B. Coronado, A.C. De Castro Lima and O. Ludwig, "Design of Automatic Meter Reading based on Zigbee" IEEE Latin America Transactions (Revista IEEE America Latina), vol.10, no.1, pp.1150-1155, Jan 2012.

[15] IEEE, Standard Definitions for the Measurement of Electric Power Quantities Under Sinusoidal, Nonsinusoidal, Balanced, or Unbalanced Conditions, IEEE Std 1459-2010, Mar 2010.

[16] A. Broshi, "Monitoring Power Quality beyond EN 50160 and IEC 610004-30" PowerAfrica '07. IEEE Power Engineering Society Conference and Exposition in Africa, pp.1,7, Jul 2007.

[17] Sawyer, D., "Nonstop monitoring [power quality]" IEE Review, vol.45, no.3, pp.126-127, Aug 1999.

[18] R.J. Romero-Troncoso, E. Cabal-Yepez, A. Garcia-Perez, R.A. OsornioRios, R. Alvarez-Salas and D. Granados-Lieberman, "Reconfigurable instrument for power quality monitoring in 3-phase power systems", IEEE International Symposium on Diagnostics for Electric Machines, Power Electronics \& Drives, pp.186,191, Sept 2011.

[19] D. De Yong, C. Reineri and F. Magnago, "Educational Software for Power Quality Analysis" Latin America Transactions, IEEE (Revista IEEE America Latina), vol.11, no.1, pp.479,485, Feb 2013.

[20] D.C. Campos Delgado and J. Luna Rivera, "Performance Study of Distributed Power Control Algorithms Under Time-Delays and Measurement Uncertainty", Latin America Transactions IEEE (Revista IEEE America Latina), vol.11, no.2, pp.690,697, Mar 2013.

[21] J.C. Gomez, J. Vaschetti, C. Coyos and C. Ibarlucea, "Distributed Generation: impact on Protections and Power Quality", Latin America Transactions IEEE (Revista IEEE America Latina), vol.11, no.1, pp.460,465, Feb 2013.

[22] A.S. Cerqueira, C.A. Duque, M.V. Ribeiro and R.M. Trindade, "Digital System for Detection and Classification of Power Quality Disturbances", Latin America Transactions IEEE (Revista IEEE America Latina), vol.4, no.5, pp.345,352, Sept 2006.

[23] M. Islam, M. Ahmad, M. Islam, A. Mitul, M. Malek and M. Rashid "Electronic energy meter with remote monitoring and billing system", 7th International Conference on Electrical \& Computer Engineering, pp. 240243, Dec 2012.

[24] B. S. Koay, S. S. Cheah, Y. H. Sng, P. H. J. Chong, P. Shum, Y. C. Tong, X. Y. Wang, Y. X. Zuo and H. W. Kuek, "Design and implementation of Bluetooth energy meter", Proceedings of the Joint Conference of the 4th International Conference on Information, Communications and Signal Processing and Pacific Rim Conference on Multimedia, pp. 1474-1477, Dec 2003.

[25] ANEEL, Resolução Normativa $\mathrm{N}^{\circ}$ 502, "Regulamenta sistemas de medição de energia elétrica de unidades consumidoras do Grupo B", 7 Aug 2012.

[26] ANEEL, Resolução Normativa No $\mathrm{N}^{\circ} 482$, "Estabelece as condições gerais para o acesso de microgeração e minigeração distribuída aos sistemas de distribuição de energia elétrica, o sistema de compensação de energia elétrica, e dá outras providências", 17 Apr 2012.

[27] R. P. S. Leao, F. L. M. Antunes, T.G.M. Lourenco, K. R. Andrade, "A Comprehensive Overview on Wind Power Integration to the Power Grid", IEEE Latin America Transactions (Revista IEEE America Latina), vol.7, no.6, pp.620,629, Dec 2009.

[28] C. Bustos, D. Watts, H. Ren, "MicroGrid Operation and Design Optimization With Synthetic Wins and Solar Resources", IEEE Latin America Transactions (Revista IEEE America Latina), vol.10, no.2, pp.1550,1562, Mar 2012.

[29] P. F. Frack, M. Martinez, M. G. Molina, P. E. Mercado, "Emulation of Synchronous Generator for Frequency Control of Smart Microgrids", IEEE Latin America Transactions (Revista IEEE America Latina), vol.11, no.1, pp.486,491, Feb 2013
[30] Depuru, S.S.S.R., Lingfeng Wang, V. Devabhaktuni and Gudi, N., "Smart meters for power grid - Challenges, issues, advantages and status", IEEE Power Systems Conference and Exposition, pp.1,7, Mar 2011.

[31] M. Chebbo, "EU SmartGrids Framework 'Electricity Networks of the future 2020 and beyond'", IEEE Power Engineering Society General Meeting, pp.1,8, Jun 2007.

[32] GOOGLE, "Google Power Meter: A google.org project", Pilot Program, 2011.

[33] C.-H. Huang, T.-T. Hsien and G.-J. Jong, "Indoor power meter combined wireless sensor network for smart grid application", 8th International Conference on Information Science and Digital Content Technology, pp. 336-339, Jun 2012.

[34] S.-W. Luan, J.-H. Teng, S.-Y. Chan and L.-C. Hwang, "Development of a smart power meter for AMI based on ZigBee communication", International Conference on Power Electronics and Drive Systems, pp. 661-665, Nov 2009

[35] H. Tan, C. Lee and V. Mok, "Automatic power meter reading system using GSM network", International Power Engineering Conference, pp. 465-469, Dec 2007

[36] P. Ovidiu and C. Gabriel, "DSP's based energy meter", 26th International Spring Seminar on Electronics Technology: Integrated Management of Electronic Materials Production, pp. 235-238, May 2003

[37] E. B. Shahid, Z. Ahmed, A. Faroqi and R.M. Navid-ur-Rehman, "Implementation of smart system based on smart grid Smart Meter and smart appliances", 2nd Iranian Conference on Smart Grids, Tehran, 2012.

[38] P. Prudhvi, D. Bhalodi, M. Manohar, V. Padidela and S. Adapa, "A smart energy meter architecture in Indian context", International Conference on Environment and Electrical Engineering, Venice, Italy, 2012.

[39] P. Tenti, P. Mattavelli and H. K. M. Paredes, "Conservative Power Theory, sequence components and accountability in smart grids", Przeglad Elektrotechniczny, v. 6, p. 30-37, 2010.

[40] P. Tenti, P. Matavelli and H. K. M. Paredes, "Conservative Power Theory, a Framework to Approach Control and Accountability Issues in Smart Microgrids”, IEEE Transactions on Power Electronics, pp. 664-673, Mar. 2011

[41] W.A. Souza, F. P. Marafão, "Desenvolvimento de sistema de aquisição para aplicações em Qualidade de Energia", Dissertação de mestrado, FEB/UNESP, Bauru, 2011.

[42] W.A. Souza, E.V. Liberado, L.C.P. da Silva, H.K.M. Paredes and F.P. Marafão, "Load Analyser using Conservative Power Theory", International School on Nonsinusoidal Currents and Compensation, Jun. 2013

[43] H. K. M. Paredes, L. C. P. da Silva, D. I. Brandão and F. P. Marafão, "Possible Shunt Compensation Strategies Based on Conservative Power Theory", Przeglad Elektrotechniczny, v. 87, p. 34-39, 2011.

[44] E. V. Liberado, W. A. Souza, H. K. M. Paredes, J. A. Pomilio and F. P. Marafão, "Design of Static VAr Compensator using Reactive Energy" International School on Nonsinusoidal Currents and Compensation, Jun 2013.

[45] P. Tenti, H. K. M. Paredes, F. Marafão and P. Mattavelli, "Accountability in Smart Microgrids Based on Conservative Power Theory", IEEE Transactions on Instrumentation and Measurement, pp. 3058-3069, Sept 2011.

[46] H. K. M. Paredes, F. P. Marafão, P. Mattavelli and P. Tenti, “Application of Conservative Power Theory to load and line characterization and revenue metering", IEEE International Workshop on Applied Measurements for Power Systems, pp. 1-6, Sept. 2012.

[47] Nota Técnica no 83/2012-SRD/ANEEL "Proposta de abertura de audiência pública para o recebimento de contribuições visando aprimorar a regulamentação acerca do fator de potência e cobrança do excedente de reativos", 2012

[48] H. K. M. Paredes, "Teoria de Potência Conservativa: Uma nova Abordagem para o Controle Cooperativo de Condicionadores de Energia e Considerações sobre Atribuição de Responsabilidades", Tese de Doutorado, FEEC/UNICAMP, Campinas, 2011.

[49] L. S. Czarnecki, "Scattered and reactive current, voltage, and power in circuits with nonsinusoidal waveforms and their compensation [power systems]", IEEE Transactions on Instrumentation and Measurement, vol.40, no.3, pp.563-574, Jun 1991.

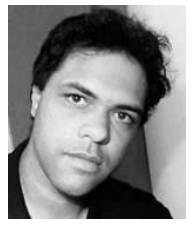

Wesley Angelino de Souza, nascido em Bauru (SP) é graduado em ciência da computação pela Faculdade de Ciências da UNESP/Bauru (2008) e tem mestrado em engenharia elétrica pela Faculdade de Engenharia pela UNESP/Bauru (2011), é atualmente doutorando em Engenharia Elétrica pela UNICAMP (a partir de 2012). No período de abril de 2013 a abril de 2014 foi pesquisador visitante na Universidade de Padova (Itália). Suas principais áreas de interesse são instrumentação, sistemas computacionais, inteligência artificial, análise e controle da qualidade de energia. 


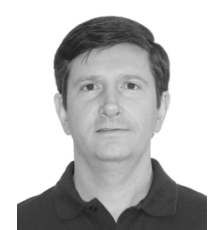

Fernando Pinhabel Marafão, nascido em José Bonifácio (SP) é graduado em engenharia elétrica pela UNESP/Bauru (1998), tem mestrado e doutorado em Engenharia Elétrica pela UNICAMP (2000 e 2004). Em 2002 e 2013 foi pesquisador visitante na Universidade de Padova (Itália) e na Colorado School of Mines (EUA), respectivamente. Desde 2005, é professor assistente doutor no Campus de Sorocaba da UNESP, onde liderou o Grupo de Automação e Sistemas Integráveis (GASI), de 2007 a 2011. Suas principais áreas de interesse são análise e controle da qualidade de energia, teorias de potência para circuitos polifásicos não lineares e, técnicas de processamento e controle digital aplicadas a sistemas de potência e eletrônica de potência. Dr. Marafão é membro da Sobraep, da SBA e do IEEE.

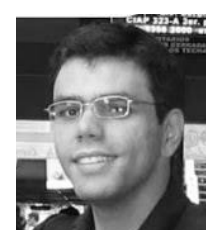

Eduardo Verri Liberado, nascido em São Paulo, é graduado em Engenharia de Controle e Automação pela Universidade Estadual Paulista - UNESP, Campus de Sorocaba (2009), com Mestrado em Engenharia Elétrica pela UNESP, Faculdade de Engenharia de Bauru (2012). Atualmente é aluno de doutorado do Programa de Pósgraduação em Engenharia Elétrica da FEEC - UNICAMP. Entre abril/2013 e março/2014 realizou estágio de Doutorado Sanduíche na Universidade de Padova (Itália). Suas principais áreas de interesse são Controle e Condicionamento de Qualidade de Energia Elétrica, Teorias de Potência e Redes Inteligentes de Energia.

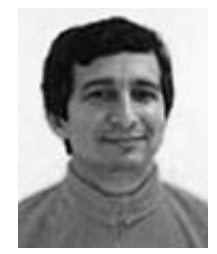

Ivando Severino Diniz, nascido em Santa Rita (PB) é Técnico em eletrotécnica pela Escola Técnica Federal da Paraíba(IFPB), graduado em Engenharia Mecânica(Centro Tecnológico) e Mestrado em Engenharia Biomédica (Centro de Ciências Médicas Hospital universitário) pela UFPB/João Pessoa. Tem doutorado em Engenharia Aeronáutica e Mecânica na área de Mecatrônica e Dinâmica de Sistemas Aeroespaciais pelo Instituto Tecnológico de Aeronáutica - ITA (2003). Desde 2004, é professor assistente doutor no Campus de Sorocaba da UNESP. Suas principais áreas de interesse são Projeto e Desenvolvimento de Sistema Embarcados (Linux, ARM ${ }^{\circledR}$ Cortex ${ }^{\mathrm{TM}}$ Microcontroller, OpenCV (Open Source Computer Vision Library)), Controle e Vibração Assistidos por Computador:CAD-CAE( NX-PLM, Easy5, ADAMS, Nastran e Patran) e Simuladores Virtuais Aplicados ao Ensino e Treinamento:(Unreal Developer's Kit, CryENGINE e Unity-3D).

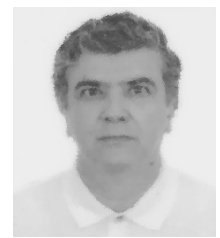

Paulo José Amaral Serni, nascido em Botucatu (SP) é engenheiro eletricista (1987) pela Faculdade de Engenharia e Tecnologia de Bauru e tem mestrado (1992) e doutorado em Engenharia Elétrica (1999) pela UNICAMP - Universidade Estadual de Campinas. É professor da UNESP - Universidade Estadual Paulista desde 1987 e docente do Curso de Engenharia de Controle e Automação - UNESP/Sorocaba desde 2012. Suas áreas de interesse são: Instrumentação e Sistemas de Medição, Eletrônica de Potência, Máquinas Elétricas e Qualidade da Energia Elétrica. 